Research Paper

\title{
Intravascular Photoacoustics for Image-Guidance and Temperature Monitoring During Plasmonic Photothermal Therapy of Atherosclerotic Plaques: A Feasibility Study
}

\author{
Doug Yeager ${ }^{1}$, Yun-Sheng Chen ${ }^{1,2}$, Silvio Litovsky ${ }^{3}$, Stanislav Emelianov¹,2 \\ 1. Department of Biomedical Engineering, University of Texas at Austin, Austin, TX, USA 78712; \\ 2. Department of Electrical and Computer Engineering, University of Texas at Austin, Austin, TX, USA 78712; \\ 3. Department of Pathology, University of Alabama Birmingham, Birmingham, AL, USA 35249.
}

\section{$\triangle$ Corresponding author: emelian@mail.utexas.edu.}

( ) Ivyspring International Publisher. This is an open-access article distributed under the terms of the Creative Commons License (http://creativecommons.org/ licenses/by-nc-nd/3.0/). Reproduction is permitted for personal, noncommercial use, provided that the article is in whole, unmodified, and properly cited.

Received: 2013.07.II; Accepted: 2013.09.12; Published: 2013.12.05

\begin{abstract}
Recently, combined intravascular ultrasound and photoacoustic (IVUS/IVPA) imaging has been demonstrated as a novel imaging modality capable of visualizing both morphology (via IVUS) and cellular/molecular composition (via IVPA) of atherosclerotic plaques, using both endogenous tissue absorbers and exogenous contrast agents. Plasmonic gold nanoparticles were previously utilized as IVPA contrast agents which co-localize with atherosclerotic plaques, particularly phagocytically active macrophages. The present work demonstrates the use of IVUS/IVPA imaging as a tool for localized temperature monitoring during laser heating. The temperature dependent change in IVPA signal intensity of silica-coated gold nanorod contrast agents absorbing within the near-infrared optical wavelength range is evaluated and shown to have a linear relationship, with a slope greater than that of endogenous tissue. A continuous wave laser was subsequently incorporated into the IVUS/IVPA integrated catheter and utilized to selectively heat the nanoparticles with simultaneous IVPA temperature monitoring. IVUS/IVPA, therefore, provides a platform for detection and temperature monitoring of atherosclerotic plaques through the selective heating of plasmonic gold nanoparticle contrast agents.
\end{abstract}

Key words: photoacoustic, atherosclerosis, gold nanorods, photothermal, temperature monitoring, intravascular.

\section{INTRODUCTION}

Coronary heart disease remains a leading cause of death throughout industrialized nations, responsible for more than 7 million deaths annually throughout the world [1]. In the United States alone, coronary heart disease accounts for approximately one in every six deaths, with coronary events occurring every 25 seconds [2]. In the majority of cases, onset of an acute coronary event is triggered by the rupture of so-called vulnerable plaques which went undetected using conventional diagnostic procedures [3]. Therefore, there remains a clinical need for improved imaging modalities and therapeutic approaches to enable differentiation and selective, local treatment of these high-risk vulnerable atherosclerotic plaques.

Current clinical imaging modalities utilized to detect the presence of atherosclerosis lack the ability to reliably differentiate plaques which are vulnerable to rupture from those which are stable. Noninvasive techniques, such as the gold standard $x$-ray angiography or coronary computed tomography, lack sufficient resolution to visualize critical vulnerable plaque characteristics, including a thin fibrous cap 
and underlying lipid-rich necrotic core with extensive macrophage accumulation. Intravascular imaging modalities, including intravascular ultrasound (IVUS) and optical coherence tomography (OCT) offer trade-offs in terms of morphological assessment capabilities based on their respective imaging depths and resolutions, but each modality is inherently limited in terms of compositional characterization due to similarities in the backscattered signal produced by different tissue types [4]. Virtual histology IVUS (VH-IVUS) seeks to expand the ability to differentiate plaque composition through analysis of the tissue-dependent changes in the frequency content of backscattered ultrasound, however the efficacy of the approach has recently been called into question $[5,6]$. Moreover, the emerging technique of diffuse reflectance near-infrared spectroscopy (NIRS) has been demonstrated to differentiate some plaque components, lipid in particular, but the modality is limited by its inability to provide a depth resolved signal [7].

Beyond the challenge of diagnosing vulnerable plaques, an effective treatment strategy has not yet been clinically adopted [8]. While preventative measures and systemic treatments are desirable as an overall policy for atherosclerosis management, localized treatment strategies are likely to remain a necessary tool for effectively treating identified vulnerable plaques. Traditional percutaneous coronary interventions, such as angioplasty and stenting have focused on mechanically restoring lumen diameter in regions with severe stenosis rather than on stabilizing plaque composition [9]. The introduction of drug eluting stents has more recently introduced a dual treatment which adds a pharmacological agent to reduce the extent of inflammation or proliferation following deployment of a stent. However, even in the case of drug eluting stents, the treatment is not specific to atherosclerotic tissue alone, and particularly not to specific biomarkers of interest within vulnerable plaques. Similarly, laser-based therapies such as excimer laser atherectomy, have also been evaluated, yet have shown only marginal clinical adoption due to increased risk of adverse effects which are largely related to a lack of specificity and undesirable damage to the vessel wall [10]. Photodynamic therapy has more recently expanded the capability of laser-based treatments and shown promise as a technique for stabilizing atherosclerotic plaques, yet has not been translated to the clinic on a large scale [11, 12].

Combined intravascular ultrasound and photoacoustic (IVUS/IVPA) imaging was recently introduced as a means of supplementing the morphological information provided by IVUS with additional capability for assessing plaque composition based on unique optical absorption spectra of tissue compo- nents or contrast agents [13]. In IVPA imaging, applied nanosecond pulsed laser energy is absorbed, inducing thermoelastic expansion and generation of photoacoustic transients within the arterial tissue which can subsequently be detected using a conventional IVUS transducer. Due to the nature of signal detection, IVPA is less sensitive to scattering than other optical modalities, enabling imaging of the full arterial thickness with optical absorption-based contrast and at comparable spatial resolution to IVUS. Integrated IVUS/IVPA catheters which couple an optical fiber to an IVUS transducer have been introduced [14] and demonstrated for imaging the distribution of endogenous absorbers within arterial plaques such as lipid [15, 16] and collagen [17, 18]. IVUS/IVPA imaging has also been expanded to include the localization of systemically injected plasmonic gold nanoparticle contrast agents. For example, spherical gold nanoparticles endocytosed by phagocytically active macrophages within atherosclerotic plaques were imaged [19]. Additionally, gold nanorods, co-localized within atherosclerotic regions, were imaged through luminal blood due to their strong optical absorbance peak within the tissue optical window [20]. The strong and tunable absorption within the near-infrared wavelength range, where endogenous tissue absorption is relatively low, makes gold nanorods attractive agents for photothermal therapy, particularly for cancer treatment applications $[21,22]$. This therapeutic approach, termed plasmonic photothermal therapy (PPTT), has more recently come under investigation as a tool for the localized treatment of atherosclerotic plaques [23, 24].

To date, PPTT for atherosclerotic plaque treatment has required a local delivery of gold nanoparticles and has been limited by an inability to monitor the delivery of therapy. IVUS/IVPA imaging offers a means to locate gold nanoparticles following systemic delivery $[19,20]$, based on unique optical absorption spectra, and to subsequently monitor temperature during PPTT, based on the temperature dependence of the generated photoacoustic signal intensity. In the case of imaging gold nanoparticles, it has been shown that conventionally utilized 3-5 ns pulsed laser illumination does not satisfy thermal confinement of the metallic structures as it does for endogenous tissue. Therefore, the general equation for induced photoacoustic pressure should be modified to reflect a dependency on the local environment of the nanoparticles, as shown in Equation 1 [25]:

$$
p_{0} \propto \eta \Gamma_{e f f} \sigma F
$$

In addition to the IVPA signal dependence on the local optical absorption cross section of the highly absorbing nanoparticle $(\sigma)$, the generated photoa- 
coustic pressure also exhibits a dependence on local optical fluence $(F)$, the heat transfer efficiency $(\eta)$ from the nanoparticle to the surroundings, and the effective Grüneisen parameter $\left(\Gamma_{\text {eff }}\right)$ of the environment immediately surrounding the absorber, which is approximated to exhibit similar temperature dependence to that of pure water in the case of water-based cells and tissue. The Grüneisen parameter is a function of the speed of sound, the thermal coefficient of volume expansion, and the isobaric specific heat capacity, all of which are temperature dependent properties. In the case of imaging gold nanoparticles in water-based tissues, this results in a linear relationship between photoacoustic signal intensity and temperature. Therefore, photoacoustics can be implemented as a tool for monitoring temperature within the local environment of optical absorbers of interest. To that end, silica-coated gold nanorods $\left(\mathrm{SiO}_{2} \mathrm{AuNR}\right)$ have been investigated as thermally stable nanosensors which, together with photoacoustic imaging, enable temperature mapping with improved sensitivity over conventional nanorods [25-27].

In the present work, we investigate IVUS/IVPA imaging as a modality for detecting and subsequently monitoring local temperature rise during selective laser heating of $\mathrm{SiO}_{2} \mathrm{AuNR}$ using a single optical fiber for both IVPA imaging and simultaneous tissue heating via continuous wave near-infrared illumination. As such, we hypothesize that IVUS/IVPA imaging can be expanded from a purely imaging-based modality to a potential theranostic platform for atherosclerotic plaque management.

\section{MATERIALS AND METHODS}

\section{Silica-Coated Gold Nanorod Preparation}

Cetyltrimethylammonium bromide (CTAB) stabilized gold nanorods were synthesized by a seed mediated growth method reported in literature [28]. Briefly, a growth solution was prepared by adding $800 \mu \mathrm{L}$ of $\mathrm{AgNO}_{3}(10 \mathrm{mM})$ and $4 \mathrm{~mL} \mathrm{HAuCl}_{4}(10 \mathrm{mM})$ to $38 \mathrm{~mL}$ of $\mathrm{CTAB}(200 \mathrm{mM})$ at $30^{\circ} \mathrm{C}$ and stirring at 500 rpm. A solution of $440 \mu \mathrm{L}$ of ascorbic acid was then added to the growth solution, resulting in a color change from yellow-orange to colorless solution. A seed solution was also prepared, consisting of $500 \mu \mathrm{L}$ of CTAB $(200 \mathrm{mM})$ and $500 \mu \mathrm{L}$ of $\mathrm{HAuCl}_{4}(0.5 \mathrm{mM})$ at $30^{\circ} \mathrm{C}$. The seed solution was vigorously stirred and 60 $\mu \mathrm{L}$ ofNaBH4 $(10 \mathrm{mM})$ was added, inducing a colorless to brown color change. After 7 minutes of aging, $96 \mu \mathrm{L}$ of the seed solution was added to the entire volume of the growth solution. The CTAB-AuNR were allowed to age over night at $30^{\circ} \mathrm{C}$ before being twice washed to remove excess $\mathrm{CTAB}$ via centrifugation at $18,000 \mathrm{rcf}$ for 40 minutes. The washed CTAB-AuNR were re-suspended in deionized water at a final peak optical density (OD) of 15, as measured by UV-Vis spectroscopy (Synergy HT, BioTek).

The CTAB was replaced with poly ethylene glycol (PEG, 2kD) by adding equal volumes of mPEG-thiol $(0.2 \mathrm{mM})$ and the OD 15 CTAB-AUNR. The mixture was sonicated for 10 minutes and allowed to react for four hours on a shaker under modest agitation. The resulting PEG-AuNR were washed via centrifuge filtration (Amicon ultra-15, Millipore) for $10 \mathrm{~min}$ at $3000 \mathrm{rcf}$ and re-suspended in water at an OD of 10. Silica coating of the PEG-AUNR was achieved by a previously reported modified Stöber method ${ }^{25}$. In short, under vigorous stirring, 1.2 $\mathrm{mL}$ of the PEG-AuNR were added to $1.8 \mathrm{~mL}$ of isopropanol, followed by the addition of $40 \mu \mathrm{L}$ of tetraethyl orthosilicate (TEOS) in isopropanol $(100 \mathrm{mM})$. A $3.8 \%$ ammonia solution in isopropanol was added to adjust the solution to a $\mathrm{pH}$ of 11 , and the solution was reacted under moderate stirring for 4 hours. The resulting silica-coated gold nanorods $\left(\mathrm{SiO}_{2} \mathrm{AuNR}\right)$ were twice washed via centrifuge filtration for $10 \mathrm{~min}$ at $1000 \mathrm{rcf}$ and re-suspended in water at the desired final OD. For in vitro cell incubation experiments, the surface of the $\mathrm{SiO}_{2} \mathrm{AuNR}$ were further modified by direct interaction of Poly-L-Lysine (1-5 kD), resulting in PLL-SiO ${ }_{2} \mathrm{AuNR}$.

\section{In vitro Loading of Macrophages with SiO2AuNR}

Mouse monocyte-derived macrophages (J774A.1) were grown to $70 \%$ confluence and incubated in phenol free DMEM F-12 media containing 5\% fetal bovine serum and PLL-SiO $\mathrm{S}_{2} \mathrm{AuNR}$ with a final nanoparticle OD of 0.5 for 24 hours at $37^{\circ} \mathrm{C}$ in $5 \% \mathrm{CO}_{2}$. The cells were then scraped and twice washed with phosphate buffered saline (PBS) via centrifugation at 300 rcf for 5 minutes to remove excess nanoparticles and finally fixed in a $10 \%$ buffered formalin solution. Two separate batches of PLL-SiO ${ }_{2}$ AuNR were utilized for the polymethylmethacrylate (PMMA) tube imaging experiment and the gelatin phantom experiment described below, with peak longitudinal absorbances of $735 \mathrm{~nm}$ and $800 \mathrm{~nm}$, respectively.

\section{Integrated IVUS/IVPA Imaging and Photo- thermal Heating System}

The combined IVUS/IVPA imaging system and the associated integrated IVUS/IVPA imaging catheter have been previously described [13, 14]. Briefly, an optical parametric oscillator (OPO) and coupled laser (PermiScan, SpectraPhysics, Inc.) providing variable wavelength optical output with 3-5 ns pulses and a spectral bandwidth of 2-3 nm and operating at a pulse repetition frequency of $10 \mathrm{~Hz}$ was utilized for genera- 
tion of photoacoustic signals. The pulsed laser optical output at wavelengths between $700 \mathrm{~nm}$ and $850 \mathrm{~nm}$ was focused into the $600 \mu \mathrm{m}$ core diameter multimode optical fiber of the integrated IVUS/IVPA imaging catheter. The distal end of the integrated catheter was angle-polished and fixed within an air-trapping cap, causing side-projecting illumination. The output energy at the distal end of the integrated catheter was maintained at approximately $0.4 \mathrm{~mJ}$ per pulse for all IVPA imaging. Distal to the end of the optical fiber, a commercially available, single element $40 \mathrm{MHz}$ IVUS transducer (Atlantis SR Pro², Boston Scientific, Inc.) was aligned with the direction of the illumination, producing overlapping light and acoustic beam profiles. During imaging, the Q-switch from the pulsed laser served as a trigger for collecting radio frequency (RF) signals from a pulser/receiver (5073PR, Olympus, Inc.) connected to the IVUS transducer of the integrated catheter. The resulting signals were collected and digitized using a 14 bit, $200 \mathrm{MHz}$ data acquisition card (CompuScope 14200 GaGe Applied Technologies, Inc.), resulting in a single IVPA A-line signal. Following a delay of $9 \mu$ sec (PDG-2515, Directed Energy Inc.), the pulser/receiver was used to trigger the transducer to enable acquisition of a conventional IVUS A-line, thus resulting in spatially co-registered IVUS and IVPA signals. The resulting acquired data was composed of spatially co-registered and temporally consecutive IVPA and IVUS signals in R-theta coordinates. A temporal separation was therefore utilized to isolate the IVPA and IVUS components of the signal. Furthermore, because IVPA imaging involves only one way travel of ultrasound, whereas IVUS signal represent a round-trip travel, the IVPA signal must be stretched by a factor of two using an interpolation algorithm in order to obtain spatially matched IVUS/IVPA images. The IVUS/IVPA data was then band-pass filtered, and envelope detected using a Hilbert transform to base-band the raw IVUS and IVPA signals. For generation of 2 dimensional images of a vessel cross section, the integrated IVUS/IVPA imaging catheter was rotated using a stepper motor (T-NM17A200, Zaber Technologies, Inc.) for a total of 256 co-registered IVUS and IVPA

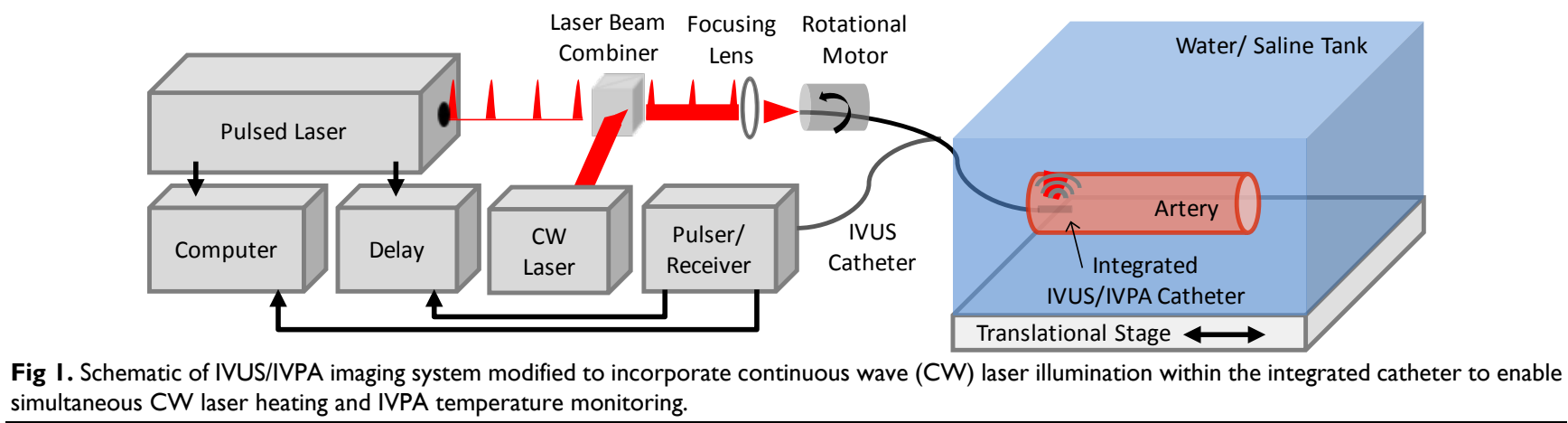

Fig I. Schematic of IVUS/IVPA imaging system modified to incorporate continuous wave $(\mathrm{CW})$ laser illumination within the integrated catheter to enable simultaneous CW laser heating and IVPA temperature monitoring. signal pairs per complete rotation. The R-theta data was scan converted from polar to Cartesian coordinates, again using an interpolation algorithm, prior to image display in order to provide conventional cross-sectional views of the image target.

To enable photothermal heating specific to the imaged vessel region, a continuous wave $(\mathrm{CW})$ diode laser (NIR DL 808-2000, Laser Lab Components Inc.) emitting at $808 \mathrm{~nm}$ was additionally focused into the optical fiber of the integrated catheter using a prism, aligned to serve as an optical beam combiner, positioned proximal to the focusing lens. CW laser output energies of $200 \mathrm{~mW}$ and $60 \mathrm{~mW}$ from the distal end of the integrated IVUS/IVPA catheter were used to assess photothermal heating and subsequent temperature dependent IVPA signal change. Reducing the output power from $200 \mathrm{~mW}$ to $60 \mathrm{~mW}$ was achieved through the addition of a neutral density filter proximal to the laser beam combiner. A schematic of the integrated IVUS/IVPA imaging and photothermal heating experimental system is presented in Figure 1.

\section{Imaging of $\mathrm{SiO}_{2}$ AuNR Inclusions}

For initial demonstration of the temperature dependence of IVPA signal intensity, IVUS/IVPA imaging was performed using an experimental setup intended to model a $\mathrm{SiO}_{2} \mathrm{AuNR}$ labeled artery. Either colloidal $\mathrm{SiO}_{2} \mathrm{AuNR}(\mathrm{OD}=20)$ or formalin fixed macrophages which had endocytosed PLL-SiO ${ }_{2} \mathrm{AuNR}$ $(\mathrm{OD}=7)$ were sealed within optically clear PMMA tubing (inner/outer diameter $=375 \mu \mathrm{m} / 500 \mu \mathrm{m}$ ) and aligned within the water tank of the IVUS/IVPA imaging system. These $\mathrm{SiO}_{2} \mathrm{AuNR}$ containing inclusions were oriented perpendicular to the axis of the integrated IVUS/IVPA imaging catheter and at a radial distance of approximately $4 \mathrm{~mm}$ from the transducer surface. The integrated catheter was fixed in a position facing the $\mathrm{SiO}_{2} \mathrm{AuNR}$ containing inclusions. The water tank was positioned on a heating plate, allowing the temperature of the entire tank and, therefore, the $\mathrm{SiO}_{2} \mathrm{AuNR}$ containing inclusions to be slowly heated and cooled, during which IVUS/IVPA signals were periodically recorded. 


\section{Phantom Preparation and Imaging}

A vessel-mimicking gelatin phantom was subsequently utilized to assess the temperature dependent IVPA signal response of more clinically-relevant tissue components. A solution containing $10 \%$ gelatin and $2 \%$ silica particles with an average diameter of 30 $\mu \mathrm{m}$, to provide optical and acoustic scattering, was molded into a hollow cylindrical shape. A piece of bovine lipid-rich tissue was implanted into the gelatin mold as the phantom solidified at $4^{\circ} \mathrm{C}$. After solidifying, an inclusion containing additional gelatin/silica solution mixed with fixed macrophages loaded with $\mathrm{SiO}_{2} \mathrm{AuNR}$ at a final OD of 2.5 was placed on the luminal surface of the phantom adjacent to the lipid-rich tissue inclusion and the phantom was again cooled at $4^{\circ} \mathrm{C}$. During IVUS/IVPA imaging, IVPA temperature monitoring was performed by slowly changing the temperature of the surrounding water tank and periodically imaging at the peak absorbance of the endocytosed nanoparticles $(735 \mathrm{~nm})$ and lipid $(1720 \mathrm{~nm})$ to differentiate the two inclusions.

\section{Ex Vivo Coronary Artery Imaging}

An atherosclerotic human right coronary artery section, obtained from a 71 year old male patient undergoing autopsy, was directly injected with a concentrated colloidal solution of $\mathrm{SiO}_{2} \mathrm{AuNR}$ on the outer surface of artery between adventitial fat and media. After 5 minutes, the artery was thoroughly washed with PBS and secured in a saline-filled tank for imaging and monitored $\mathrm{CW}$ photothermal heating. IVUS/IVPA imaging was performed at $808 \mathrm{~nm}$ to create a cross-sectional image revealing the artery morphology and location of the nanoparticle inclusion. The integrated catheter was then oriented to detect a region of high IVPA signal from the nanoparticles and temperature monitoring along a single A-line was performed by adjusting the temperature of the saline tank as well as by inducing CW photothermal heating. A separate location from the cross-section which did not contain the $\mathrm{SiO}_{2} \mathrm{AuNR}$ inclusion was also imaged as a control.

\section{FLIR Thermal Imaging}

A forward looking infrared (FLIR) thermal camera was utilized to confirm the extent and specificity of $\mathrm{CW}$ heating of $\mathrm{SiO}_{2} \mathrm{AuNR}$ relative to background tissue of the human coronary artery sample. The FLIR thermal camera is capable of providing only surface temperature measurements, so the experimental set-up was modified following IVPA monitored heating to enable confirmation of heating using the thermal camera. Specifically, the coronary artery remained fixed within the saline tank, but the saline level was reduced to the surface of the artery. The optical fiber from the integrated IVUS/IVPA catheter was then positioned outside of the artery and focused onto a region of interest of the coronary artery, including the $\mathrm{SiO}_{2} \mathrm{AuNR}$ inclusion and a control region without $\mathrm{SiO}_{2} \mathrm{AuNR}$. The FLIR thermal camera (FLIR A325sc, FLIR Systems, Inc.) was focused on the saline tank, and temperature profiles of regions of interest were recorded for 90 seconds (ExaminIR software, FLIR Systems, Inc.), beginning at approximately the same time as the start of the application of CW laser. $\mathrm{CW}$ laser heating was applied for approximately the first 45 seconds, after which the CW laser was turned off, allowing the artery to cool. The temporal temperature profiles were obtained from a region of interest within the $\mathrm{CW}$ laser spot on the arterial surface for three different data sets; the arterial region containing the $\mathrm{SiO}_{2} \mathrm{AuNR}$ inclusion which was illuminated with $200 \mathrm{~mW}$ laser output power, the arterial region containing the $\mathrm{SiO}_{2} \mathrm{AuNR}$ inclusion which was illuminated with $60 \mathrm{~mW}$ laser output power, and a control region on the arterial surface which did not contain $\mathrm{SiO}_{2} \mathrm{AuNR}$ but which was illuminated with $200 \mathrm{~mW}$ laser output power. Additionally, for each case, thermal images were taken after a time of 45 seconds of $\mathrm{CW}$ laser heating, just prior to the $\mathrm{CW}$ laser being turned off, when the laser-induced heating is expected to have been at its maximum. The acquired raw thermal data was then converted to surface temperature $\left({ }^{\circ} \mathrm{C}\right)$ using a black body temperature calibration curve.

\section{RESULTS}

The linearity of IVPA signal intensity change with temperature was first verified through a set of experiments in which the environment surrounding the imaging target was incrementally heated or cooled. Imaging targets ranged from $\mathrm{SiO}_{2} \mathrm{AuNR}$ at varying concentrations and macrophages which had phagocytosed PLL-SiO ${ }_{2} \mathrm{AuNR}$ to vessel-mimicking phantoms and ex vivo tissue labeled with $\mathrm{SiO}_{2} \mathrm{AuNR}$. The experimental set-up was subsequently modified to incorporate the addition of a continuous wave laser emitting at a wavelength of $808 \mathrm{~nm}$ and coupled into the same optical fiber of the integrated IVUS/IVPA imaging catheter that was used to deliver the nanosecond pulsed laser illumination necessary for IVPA signal generation. The ability to induce selective heating of the $\mathrm{SiO}_{2} \mathrm{AuNR}$ with the $\mathrm{CW}$ laser and to monitor the resulting targeted heating using IVUS/IVPA imaging was then investigated in a series of similar experiments.

Figure 2A demonstrates the change in IVPA signal intensity versus temperature. The presented data was recorded during cooling (blue) and subsequent reheating (red) of the water tank containing 
$\mathrm{SiO}_{2} \mathrm{AuNR}$ within PMMA tubing at an OD of 20 over a temperature range from approximately $29^{\circ} \mathrm{C}$ to $43^{\circ} \mathrm{C}$. The monotonic change in measured IVPA signal intensity demonstrates the linearity and reproducibility of IVPA temperature response. Similarly, Figure $2 \mathrm{~B}$ presents the percent change in IVPA signal intensity as a function of change in temperature for both the OD $20 \mathrm{SiO}_{2} \mathrm{AuNR}$ (green) and a solution containing formalin fixed J774A.1 macrophages which had previously phagocytosed PLL-SiO ${ }_{2} \mathrm{AUNR}$ with a final OD of 7 . The linearity of IVPA temperature response is again confirmed for $\mathrm{SiO}_{2} \mathrm{AuNR}$, including both the colloidal particles and those within endosomes. Additionally, a change in IVPA signal of approximately $50 \%$ was measured for the OD $20 \mathrm{SiO}_{2} \mathrm{AuNR}$ over a $10^{\circ} \mathrm{C}$ temperature change versus a $90 \%$ change in IVPA signal from $\mathrm{SiO}_{2} \mathrm{AuNR}$ within macrophages over the same range. This variation in temperature sensitivity is indicative of a variation in the effective Grüneisen parameter of solutions containing colloidal nanoparticles versus those aggregated within endosomes.

Temperature dependent IVPA signal changes were further investigated using a $10 \%$ gelatin tissue-mimicking phantom containing two inclusions applicable to atherosclerotic plaques, one with fresh bovine lipid-rich tissue and the other with formalin-fixed macrophages which had endocytosed PLL-SiO ${ }_{2} \mathrm{AuNR}$ with a longitudinal peak absorbance of $735 \mathrm{~nm}$. Figure 3 demonstrates the temperature dependence of the IVPA signal intensity detected within the two inclusions. Imaging performed at the $\mathrm{SiO}_{2} \mathrm{AuNR}$ absorbance peak of $735 \mathrm{~nm}$ with the phantom at $11^{\circ} \mathrm{C}$ (Fig. $3 \mathrm{~A}$ ) results in a significantly lower signal than that detected at $26^{\circ} \mathrm{C}$ (Fig. 3B). Likewise, imaging of the lipid-rich tissue inclusion, performed at a lipid absorbance peak located at 1720 $\mathrm{nm}$ which has been previously utilized for IVPA imaging of lipid-rich plaques, reveals a similar trend over the same temperature range (Fig.3C, D). Note that the lipid-rich inclusion also causes a significant shadowing effect in the IVUS image within the 1-2 o'clock region. All IVPA images are displayed on the same linear scale, overlaying IVUS images displayed on $40 \mathrm{~dB}$ dynamic range.

Figure 3E provides a plot of the IVPA signal intensity with varying temperature for three regions, the $\mathrm{SiO}_{2} \mathrm{AuNR}$ loaded macrophage inclusion (12 o'clock), the adjacent lipid-rich tissue inclusion (1 o'clock), and a control region from within the gelatin phantom (9 o'clock). Data for the $\mathrm{SiO}_{2} \mathrm{AuNR}$ loaded macrophage inclusion and the control region were obtained from IVPA imaging at $735 \mathrm{~nm}$, while the data obtained for the lipid-rich tissue region was obtained from IVPA imaging at $1720 \mathrm{~nm}$. The plot reveals a linear increase in IVPA signal with temperature for all three regions, but a significantly greater slope for the $\mathrm{SiO}_{2} \mathrm{AuNR}$ containing inclusion. An increase in temperature of $10^{\circ} \mathrm{C}$ resulted in corresponding percent increases in IVPA signal of $98.4 \%$, $20.5 \%$, and $4.4 \%$ for the $\mathrm{SiO}_{2} \mathrm{AuNR}$ loaded macrophage inclusion, lipid-rich tissue inclusion, and control region, respectively. This large difference in temperature-dependent signal change further supports the use of $\mathrm{SiO}_{2} \mathrm{AuNR}$ as sensitive thermal sensors for IVPA imaging.
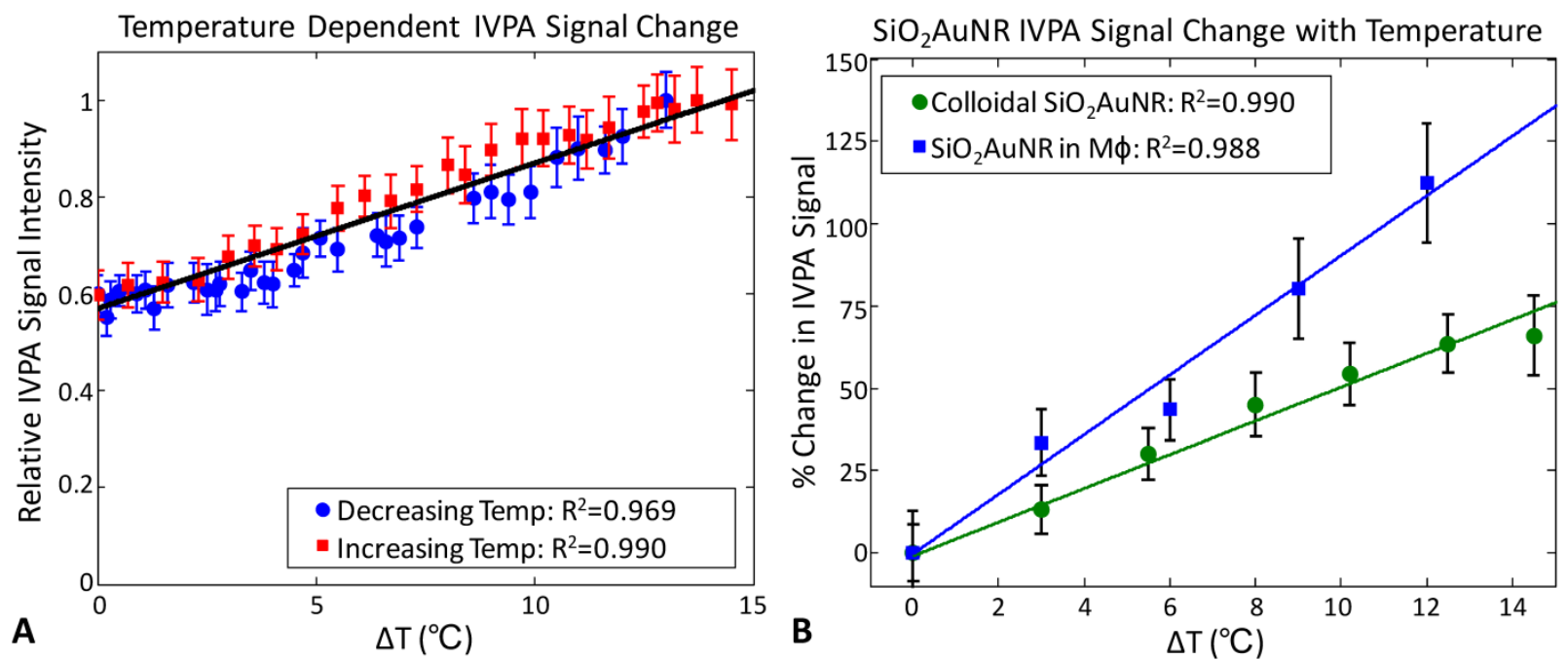

Fig 2. Temperature dependence of IVPA signal from $\mathrm{SiO}_{2} \mathrm{AuNR}$ within PMMA tubing. (A) Peak IVPA signals during cooling (blue) and subsequent reheating (red) of a water tank containing an inclusion of $O D=20 \mathrm{SiO}_{2} A u N R$. (B) Percent change in peak IVPA signal versus change in temperature for OD 20 $\mathrm{SiO}_{2} \mathrm{AUNR}$ (green) and $\mathrm{SiO}_{2} \mathrm{AuNR}$ phagocytosed by macrophages (blue) at a final concentration of $\mathrm{OD}=7$. Data represents the mean and standard deviation from 100 IVPA measurements. 

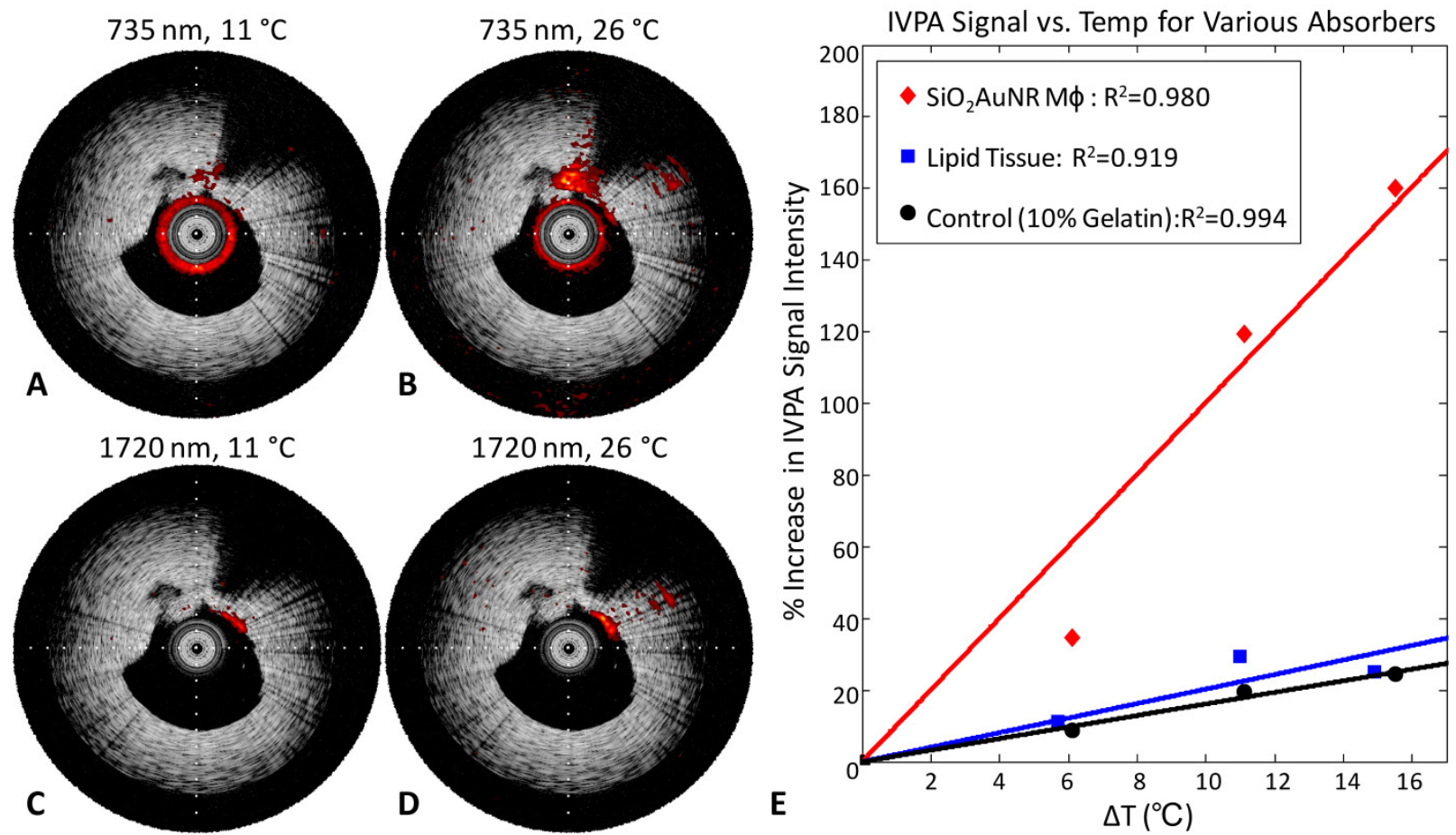

Fig 3. IVUS/IVPA imaging of a tissue-mimicking phantom with lipid and $\mathrm{SiO}_{2} \mathrm{AuNR}$ inclusions. IVUS/IVPA image of an inclusion with macrophages labeled with PLL-SiO ${ }_{2} \mathrm{AuNR}$, acquired at an imaging wavelength of $735 \mathrm{~nm}$, at temperatures of (A) $11^{\circ} \mathrm{C}$ and (B) $26^{\circ} \mathrm{C}$. IVUS/IVPA image of an inclusion with lipid-rich tissue, acquired at an imaging wavelength of $1720 \mathrm{~nm}$, at temperatures of (C) $11^{\circ} \mathrm{C}$ and (D) $26^{\circ} \mathrm{C}$. Tick marks on IVUS/IVPA images are spaced at $1 \mathrm{~mm}$. Plot of the temperature dependence of IVPA signal intensity for the PLL-SiO ${ }_{2} \mathrm{AuNR}$ loaded macrophages, lipid-rich tissue, and a control region within the phantom $(E)$.
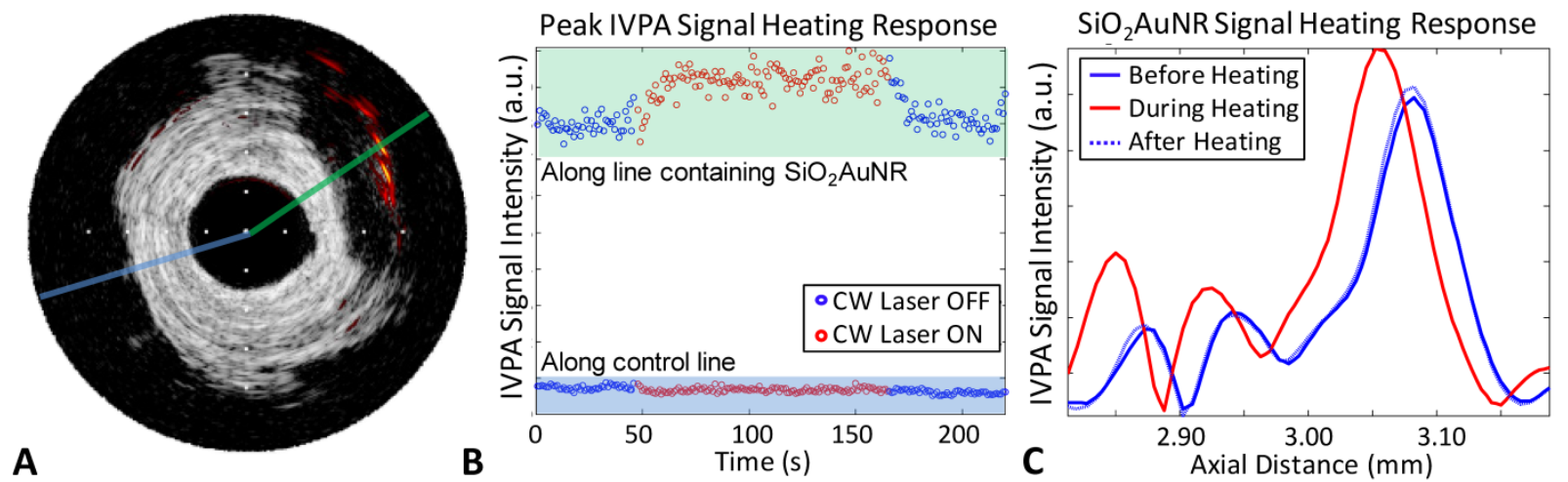

Fig 4. Ex-vivo demonstration of IVPA monitored $\mathrm{CW}$ laser induced heating of $\mathrm{SiO}_{2} \mathrm{AUNR}$ within a human coronary artery. (A) IVUS/IVPA image obtained at $808 \mathrm{~nm}$ revealing the location of $\mathrm{SiO}_{2} \mathrm{AuNR}$ at 2 o'clock. Tick marks are spaced at I mm. (B) Maximum IVPA signal along the $\mathrm{SiO}_{2} \mathrm{AuNR}$ containing and control lines, indicated in A, versus time. The CW laser initially off (blue), then turned on to induce heating (red) and back off (blue). (C) Section of the IVPA A-line shown in green in part $\mathrm{A}$ which containing the $\mathrm{SiO}_{2} \mathrm{AuNR}$ inclusion, revealing the reversibly induced increase and shift in peak IVPA intensity during $\mathrm{CW}$ laser heating.

Next, the IVUS/IVPA system was modified to incorporate a near-infrared emitting CW laser, as indicating in Figure 1. The CW laser was utilized as a means of selectively heating $\mathrm{SiO}_{2} \mathrm{AuNR}$, capitalizing on the overlap of the emission wavelength of $808 \mathrm{~nm}$ with the nanoparticles' longitudinal peak absorbance and the relatively low blood and tissue absorption within the tissue optical window. $\mathrm{SiO}_{2} \mathrm{AuNR}$ injected into the adventitial fat of a human right coronary artery were imaged $e x$ vivo and utilized to demonstrate the ability to induce and monitor $\mathrm{CW}$ laser heating, Figure 4. The IVUS/IVPA image reveals the presence of the $\mathrm{SiO}_{2} \mathrm{AuNR}$ region at approximately 2 o'clock (Fig. 4A). CW laser heating with IVPA monitoring was subsequently performed along the two lines indicated, without rotation of the integrated catheter. Figure $4 \mathrm{~B}$ reveals the peak IVPA signal intensity along the indicated lines, with the $\mathrm{SiO}_{2} \mathrm{AuNR}$ containing line (green) on the top, and the control line (blue) on the bottom. As expected from 
the IVUS/IVPA image in Figure 4A, the control line has a significantly lower peak IVPA signal intensity than that of the $\mathrm{SiO}_{2} \mathrm{AuNR}$ containing line. Additionally, an increase in the peak IVPA singal for the line containing $\mathrm{SiO}_{2} \mathrm{AuNR}$ can be seen while the $\mathrm{CW}$ laser is applied, indicative of heating. Conversely, no similar increase is seen from the region without $\mathrm{SiO}_{2} \mathrm{AuNR}$. Further evidence of $\mathrm{SiO}_{2} \mathrm{AuNR}$ heating can be seen in Figure 4C, which provides a plot of the $\mathrm{SiO}_{2} \mathrm{AuNR}$ containing line from representative times while the CW laser was off (blue) and on (red). The peak IVPA signal obtained from the $\mathrm{SiO}_{2} \mathrm{AuNR}$ region reveals a reversible increase and proximal shift (decrease in the temporal occurance of the peak)while CW heating is applied.

The consistency of the IVPA signal change with respect to both changing saline environment temperature and $\mathrm{CW}$ laser induced heating of a single location containing $\mathrm{SiO}_{2} \mathrm{AuNR}$ within the human coronary artery is demonstrated in Figure 5A. The percent change in IVPA signal with respect to change in the saline tank temperature in the absence of any $\mathrm{CW}$ laser heating is provided in black, with a $10^{\circ} \mathrm{C}$ temperature rise resulting in an $83.6 \%$ change in measured IVPA signal intensity. At varying saline tank temperatures, the $\mathrm{CW}$ laser was also briefly turned on to produce additional heating of the $\mathrm{SiO}_{2} \mathrm{AuNR}$ using distinct output energies of either $60 \mathrm{~mW}$ (orange) or $200 \mathrm{~mW}$ (blue). Each of the plotted lines represents the total percent IVPA signal increase from the baseline saline tank temperature, including both the increased temperature of the saline environment and the locally induced temperature rise caused by $\mathrm{CW}$ heating, if applied. By inspection of the percent change in IVPA signal induced by the CW laser, it is evident that the IVPA monitored heating of the $\mathrm{SiO}_{2} \mathrm{AuNR}$ amounted to average local temperature rises of approximately $3.0^{\circ} \mathrm{C}(21.1 \%$ increase in IVPA signal intensity) and $7.1^{\circ} \mathrm{C}(58.8 \%$ increase in IVPA signal intensity) over the baseline saline and arterial temperature (black) for the $60 \mathrm{~mW}$ and $200 \mathrm{~mW}$ output energies, respectively.

The FLIR images and temperature profiles presented in Figure 5B further confirm the extent of CW laser induced heating and the specificity of heating to the region of the human coronary artery containing $\mathrm{SiO}_{2} \mathrm{AuNR}$. Each of the FLIR thermal images shown were generated following approximately 45 seconds of CW laser heating. The optical fiber, seen on the right of each image, was focused on a region of interest, either the $\mathrm{SiO}_{2} \mathrm{AuNR}$ inclusion (top and middle) or a control region (bottom) of the human coronary artery. Illumination of the $\mathrm{SiO}_{2} \mathrm{AuNR}$ inclusion with optical fiber output powers of $200 \mathrm{~mW}$ and $60 \mathrm{~mW}$ resulted in maximum temperature rises of $6.9^{\circ} \mathrm{C}$ (top) and $2.8^{\circ} \mathrm{C}$ (middle), respectively, as demonstrated by the temporal profile of the region of interest from the thermal image which corresponds to the surface of the $\mathrm{SiO}_{2} \mathrm{AuNR}$ inclusion. These values are in good agreement with the measured temperature rise using the integrated IVPA imaging catheter (Fig. 5A). The slight differences observed between the IVPA monitored temperature rise and that measured using FLIR imaging are likely due to the required modifications in their respective experimental set-ups and the fact that FLIR thermal imaging yields only a superficial temperature measurement. The elapsed time which was required to reach steady-state temperature during and following CW laser heating which was observed in the case of $200 \mathrm{~mW}$ power output (top) is also in agreement with the temporal rise and fall profiles of the recorded IVPA signal intensity (Fig. 4B). Additional FLIR thermal imaging performed with an optical fiber output power of $200 \mathrm{~mW}$ illuminating a region of the coronary artery which did not contain $\mathrm{SiO}_{2} \mathrm{AuNR}$ reveals a temperature rise of less than $1.5^{\circ} \mathrm{C}$ (bottom), further confirming that background tissue heating is minimized by utilizing $\mathrm{CW}$ laser heating of $\mathrm{SiO}_{2} \mathrm{AuNR}$ strongly absorbing within the near-infrared optical wavelength range.

\section{DISCUSSION}

The linearity of IVPA signal intensity dependence on temperature was demonstrated on both colloidal particles and those aggregated within macrophage endosomes. Such a relationship was anticipated based on the sensitivity of the Grüneisen parameter to variations in temperature. Therefore, provided that there is adequate absorption to produce a photoacoustic pressure wave, IVPA provides a mechanism for measuring temperature variations within the local environment surrounding the absorbers. AuNR were previously found to preferentially accumulate within plaques of atherosclerotic rabbit models following systemic injection, and the ability to localize the nanoparticles using spectroscopic IVUS/IVPA imaging, including imaging in the presence of luminal blood, was recently demonstrated [20]. Herein, the ability to further utilize such a delivery method and the resulting AuNR contrast over surrounding blood and endogenous tissue within the near-infrared wavelength range for selective CW laser heating and IVPA temperature monitoring was introduced. By measuring the percent change in IVPA signal intensity versus the change in temperature, it is possible to assess local temperature rise independent of the local pulsed laser fluence. This method, therefore, effectively self-normalizes based on the change in photoacoustic signal, accounting for possible depth-dependent variations in both the local laser fluence and integrated IVUS/IVPA catheter sensitivity. 


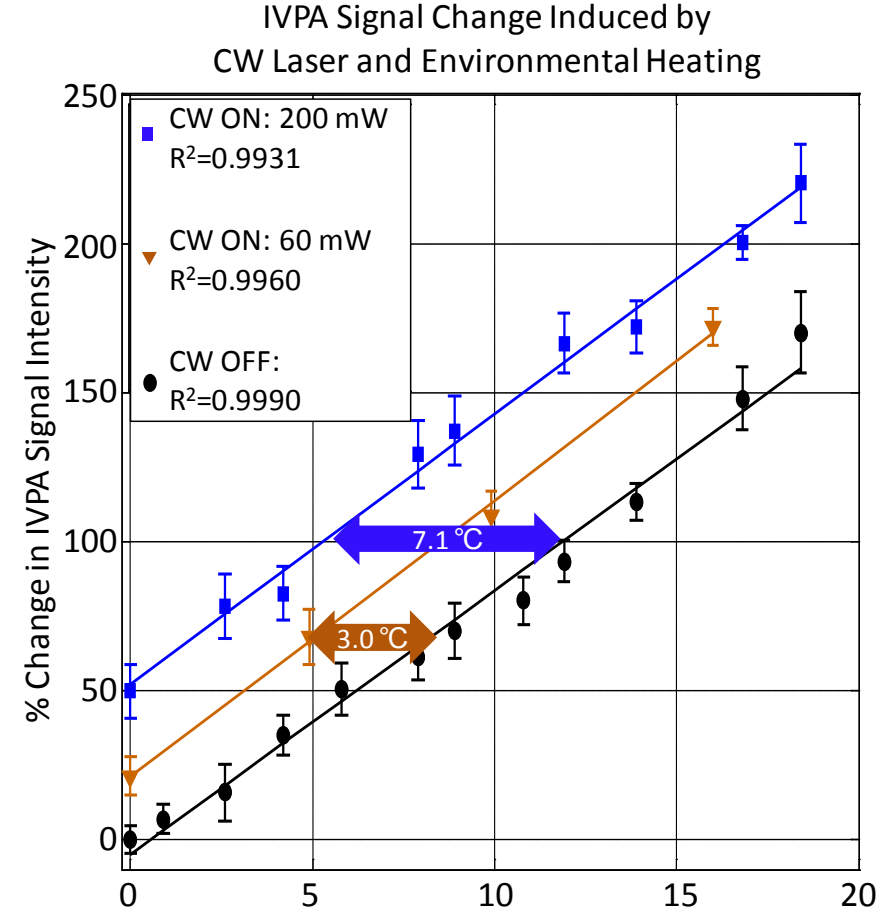

A

Temperature Increase of Saline Tank $\left({ }^{\circ} \mathrm{C}\right)$

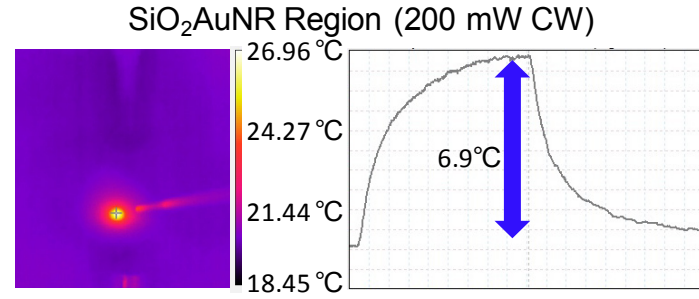

$\mathrm{SiO}_{2}$ AuNR Region (60 mW CW)

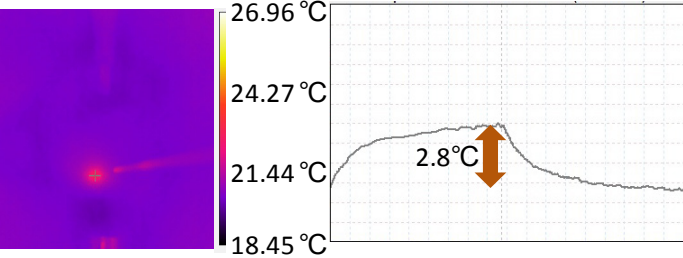

Control Region (200 mW CW)

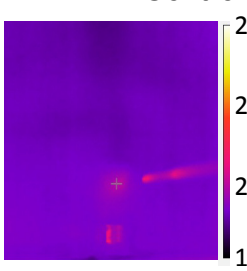

B

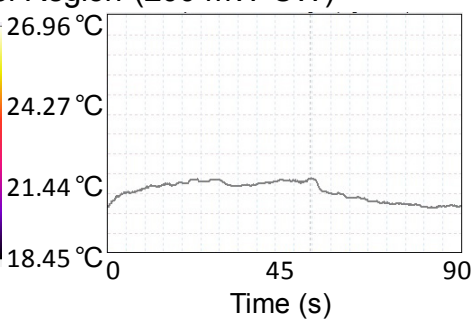

Fig 5. Confirmation of IVPA temperature monitoring during heating of $\mathrm{SiO}_{2} \mathrm{AuNR}$ on a human right coronary artery ex-vivo. (A) The percent IVPA signal change versus relative baseline temperature are plotted in the absence of $C W$ laser heating (black), and following $C W$ laser heating of the SiO ${ }_{2} A u N R$ inclusion using output energies of $60 \mathrm{~mW}$ (orange) and $200 \mathrm{~mW}$ (blue) from the integrated IVUS/IVPA imaging catheter. (B) FLIR thermal images and temporal profiles of surface temperature rise of the $\mathrm{SiO}_{2} \mathrm{AuNR}$ inclusion exposed to $200 \mathrm{~mW}$ (top) and $60 \mathrm{~mW}$ (middle) $\mathrm{CW}$ laser heating and a control region of the artery exposed to $200 \mathrm{~mW} \mathrm{CW}$ laser heating (bottom).

In addition to the IVPA monitored local temperature rise, the IVUS signal can also be used to monitor the cumulative heating along a given line due to the temperature dependence of the speed of sound, which affects not only the photoacoustic signal amplitude based on its inclusion as part of the Grüneisen parameter, but also the acoustic time of flight. This effect is demonstrated in Figure 4C and 4D in which proximal shifts (i.e., reduced acoustic time of flight) of both the IVPA and IVUS peaks are noticeable as a result of the increased speed of sound during $\mathrm{CW}$ laser heating (red lines). Additionally, a qualitative comparison of the extent of IVUS peak signal shifts at the location of the $\mathrm{SiO}_{2} \mathrm{AuNR}$ inclusion, approximately $6.1 \mathrm{~mm}$, and at the luminal boundary of the coronary artery, approximately $3 \mathrm{~mm}$, suggests that the use of a near-infrared wavelength laser causes minimal diffuse heating at the lumen border. For uniform heating, the ratio of the signal shift to the distance from the transducer would be expected to remain constant, however the ratio is greater at the location of the nanoparticle inclusion than it is at the tissue luminal surface. This further confirms the ability to selectively heat the $\mathrm{SiO}_{2} \mathrm{AuNR}$ with minimal nonspecific vascular heating.

Interestingly, results presented in Figures 2, 3, and 5 provide evidence that the slope of the IVPA signal change with temperature is dependent on more than the net $\mathrm{SiO}_{2} \mathrm{AuNR}$ density alone. This is particularly evident in Figure 2B, in which cells containing the nanoparticles within endosomes exhibit a significantly greater percent IVPA signal change with temperature change than do colloidal particles with a higher net OD. This apparent discrepancy, along with variability in the measured percent IVPA signal change per $10^{\circ} \mathrm{C}$ temperature rise between experiments, is presumably due to variations in the aggregation state and distribution of the $\mathrm{SiO}_{2} \mathrm{AuNR}$. In the case of endocyosed $\mathrm{SiO}_{2} \mathrm{AuNR}$, groups of particles are expected to be closely packed, resulting in an enhanced efficiency of photoacoustic signal generation for a given change in the local temperature based on a greater temperature sensitivity of the effective Grüneisen parameter. Given that variations in both $\mathrm{SiO}_{2} \mathrm{AuNR}$ concentration and distribution can influence a perceived temperature rise based on a measured IVPA signal change, future work will seek to establish an understanding of the variability of $\mathrm{CW}$ induced heating as a function of nanoparticle concentration within cells, followed by a longitudinal study to validate the therapeutic efficacy of IVUS/ IVPA-monitored PPTT of atherosclerotic plaques in 
vivo. These future studies will seek to evaluate the minimum temperature sensitivity of the proposed approach based on the extent of $\mathrm{SiO}_{2} \mathrm{AuNR}$ loading which can be achieved within atherosclerotic plaques following systemic injection, as well as to establish a desirable thermal dose for optimal PPTT therapeutic efficacy.

\section{CONCLUSIONS}

It has been shown that IVPA imaging can be used to monitor temperature change of the local environment surrounding $\mathrm{SiO}_{2} \mathrm{AuNR}$ based on the linear relationship between photoacoustic pressure generation and the temperature dependent Grüneisen parameter, and that such temperature change can be effectively achieved by incorporating near-infrared wavelength emitting $\mathrm{CW}$ laser illumination into the integrated IVUS/IVPA catheter's optical fiber to induce preferential heating of $\mathrm{SiO}_{2} \mathrm{AuNR}$ contrast agents. IVUS/IVPA can therefore be used as a theranostic platform to first detect the presence of systemically injected gold nanoparticles within atherosclerotic plaques, as has been previously demonstrated, followed by subsequent IVPA-monitored, selective plasmonic photothermal heating delivered through the integrated imaging catheter. This method offers a potential solution to limitations of other intravascular laser based therapies which suffer from both a lack of specificity in the induced thermal damage and an inability to monitor the delivered thermal dose. The tendency of gold nanoparticles to be preferentially endocytosed by macrophages further support the adoption of this contrast enhanced technique as a cellular-specific therapeutic strategy for atherosclerotic plaques, and the future conjugation of targeting moieties to the $\mathrm{SiO}_{2} \mathrm{AuNR}$ surface may provide a means of cellular/molecular specificity beyond macrophages.

\section{ABBREVIATIONS}

AuNR: Gold Nanorods; IVPA: intravascular photoacoustic; IVUS: intravascular ultrasound; OD: optical density; PBS: phosphate buffered saline; PEG: polyethylene glycol; PLL: poly-l-lysine; PMMA: polymethylmethacrylate; PPTT: plasmonic photothermal therapy; $\mathrm{SiO}_{2} \mathrm{AuNR}$ : silica-coated gold nanorods;

\section{ACKNOWLEDGEMENTS}

The authors would like to thank Dr. Andrei Karpiouk from the University of Texas at Austin for the fabrication of the integrated IVUS/IVPA catheter and technical advice for coupling the additional laser source. This work was supported in part by the $\mathrm{Na}-$ tional Institutes of Health grant numbers CA149740,
HL096981 and 1F31AG042247.

\section{COMPETING INTERESTS}

The authors have declared that no competing interest exists.

\section{REFERENCES}

1. Mendis S PP, Norrving B. Global Atlas on Cardiovascular Disease Prevention and Control. Geneva, Switzerland: WHO, World Heart Federation, World Stroke Organization; 2011.

2. Roger VrL, Go AS, Lloyd-Jones DM, Benjamin EJ, Berry JD, Borden WB, et al. Heart Disease and Stroke Statistics-2012 Update: A Report From the American Heart Association. Circulation. 2012; 125: e2-e220.

3. Kolodgie FD, Virmani R, Burke AP, Farb A, Weber DK, Kutys R, et al. Pathologic assessment of the vulnerable human coronary plaque. Heart. 2004; 90: 1385-91.

4. MacNeill BD, Lowe HC, Takano M, Fuster V, Jang I-K. Intravascular Modalities for Detection of Vulnerable Plaque: Current Status. Arterioscler Thromb Vasc Biol. 2003; 23: 1333-42.

5. Thim T, Hagensen MK, Wallace-Bradley D, Granada JF, Kaluza GL, Drouet L, et al. Unreliable Assessment of Necrotic Core by Virtual Histology Intravascular Ultrasound in Porcine Coronary Artery Disease. Circ Cardiovasc Imaging. 2010; 3: 384-91.

6. Obaid DR, Calvert PA, McNab D, West NEJ, Bennett MR. Identification of Coronary Plaque Sub-Types Using Virtual Histology Intravascular Ultrasound Is Affected by Inter-Observer Variability and Differences in Plaque Definitions. Circ Cardiovasc Imaging. 2011; 5: 86-93.

7. Gardner CM, Tan H, Hull EL, Lisauskas JB, Sum ST, Meese TM, et al. Detection of Lipid Core Coronary Plaques in Autopsy Specimens With a Novel Catheter-Based Near-Infrared Spectroscopy System. JACC Cardiovasc Imaging. 2008; 1: 638-48.

8. Naghavi M, Falk E, Hecht HS, Jamieson MJ, Kaul S, Berman D, et al. From Vulnerable Plaque to Vulnerable Patient Part III: Executive Summary of the Screening for Heart Attack Prevention and Education (SHAPE) Task Force Report. Am J Cardiol. 2006; 98: 2-15.

9. Smith SC, Dove JT, Jacobs AK, Kennedy JW, Kereiakes D, Kern MJ, et al. ACC/AHA Guidelines for Percutaneous Coronary Intervention (Revision of the 1993 PTCA Guidelines) Executive Summary: A Report of the American College of Cardiology/American Heart Association Task Force on Practice Guidelines (Committee to Revise the 1993 Guidelines for Percutaneous Transluminal Coronary Angioplasty) Endorsed by the Society for Cardiac Angiography and Interventions. Circulation. 2001; 103: 3019-41.

10. Baumbach A, Bittl JA, Fleck E, Geschwind HJ, Sanborn TA, Tcheng JE, et al. Acute complications of excimer laser coronary angioplasty: A detailed analysis of multicenter results. J Am Coll Cardiol. 1994; 23: 1305-13.

11. Waksman R, McEwan PE, Moore TI, Pakala R, Kolodgie FD, Hellinga DG, et al. PhotoPoint Photodynamic Therapy Promotes Stabilization of Atherosclerotic Plaques and Inhibits Plaque Progression. J Am Coll Cardiol. 2008; 52: 1024-32.

12. Rockson SG, Kramer P, Razavi M, Szuba A, Filardo S, Fitzgerald P, et al. Photoangioplasty for Human Peripheral Atherosclerosis: Results of a Phase I Trial of Photodynamic Therapy With Motexafin Lutetium (Antrin). Circulation. 2000; 102: 2322-4.

13. S. Sethuraman SRA, J. H. Amirian, R. W. Smalling, and S. Y. Emelianov. Intravascular photoacoustic imaging using an IVUS imaging catheter. IEEE Trans Ultrason Ferroelectr Freq Control. 2007; 54: 978-86.

14. Andrei BK, Bo W, Stanislav YE. Development of a catheter for combined intravascular ultrasound and photoacoustic imaging. Rev Sci Instrum. 2010; 81: 014901.

15. Wang B, Su JL, Amirian J, Litovsky SH, Smalling R, Emelianov S. Detection of lipid in atherosclerotic vessels using ultrasound-guided spectroscopic intravascular photoacoustic imaging. Opt Express. 2010; 18: 4889-97.

16. Jansen K, van der Steen AFW, van Beusekom HMM, Oosterhuis JW, van Soest G. Intravascular photoacoustic imaging of human coronary atherosclerosis. Opt Lett. 2011; 36: 597-9.

17. Sethuraman S, Amirian JH, Litovsky SH, Smalling RW, Emelianov SY. Ex vivo Characterization of Atherosclerosis using Intravascular Photoacoustic Imaging. Opt Express. 2007; 15: 16657-66.

18. Wang P, Wang P, Wang H, Cheng J. Mapping lipid and collagen by multispectral photoacoustic imaging of chemical bond vibration. J Biomed Opt. 2012; 17: 96010-1.

19. Wang B, Yantsen E, Larson T, Karpiouk AB, Sethuraman S, Su JL, et al. Plasmonic Intravascular Photoacoustic Imaging for Detection of Macrophages in Atherosclerotic Plaques. Nano Lett. 2008; 9: 2212-7.

20. Yeager D, Karpiouk A, Wang B, Amirian J, Sokolov K, Smalling R, et al. Intravascular photoacoustic imaging of exogenously labeled atherosclerotic plaque through luminal blood. J Biomed Opt. 2012; 17: 106016.

21. Alkilany AM, Thompson LB, Boulos SP, Sisco PN, Murphy CJ. Gold nanorods: Their potential for photothermal therapeutics and drug delivery, tempered by the complexity of their biological interactions. Adv Drug Deliv Rev. 2012; 64: $190-9$ 
22. Zhang Z WJ, Chen C. Gold Nanorods Based Platforms for Light-Mediated Theranostics. Theranostics. 2013; 3: 223-38.

23. Kharlamov AN, Gabinsky JL. Plasmonic photothermic and stem cell therapy of atherosclerotic plaque as a novel nanotool for angioplasty and artery remodeling. Rejuvenation Res. 2012; 15: 222-30.

24. Kharlamov AN, Tyurnina AE, Veselova VS, Novoselova OS, Filatova AS, Kovtun OP, et al. Plasmonics for Treatment of Atherosclerosis: Results of NANOM-FIM Trial. J Nanomed Nanotechol. 2013; 4: 160.

25. Chen Y-S, Frey W, Kim S, Kruizinga P, Homan K, Emelianov S. Silica-Coated Gold Nanorods as Photoacoustic Signal Nanoamplifiers. Nano Lett. 2011; 11: 348-54.

26. Chen Y-S, Frey W, Kim S, Homan K, Kruizinga P, Sokolov K, et al. Enhanced thermal stability of silica-coated gold nanorods for photoacoustic imaging and image-guided therapy. Opt Express. 2010; 18: 8867-78.

27. Chen Y-S, Frey W, Walker C, Aglyamov S, Emelianov S. Sensitivity enhanced nanothermal sensors for photoacoustic temperature mapping. J Biophotonics. 2013; 6: 534-42.

28. Jana NR, Gearheart L, Murphy CJ. Seed-Mediated Growth Approach for Shape-Controlled Synthesis of Spheroidal and Rod-like Gold Nanoparticles Using a Surfactant Template. Adv Mater. 2001; 13: 1389-93. 